\title{
PHOTOGRAPHIC PHOTOMETRY OF V 1057 CYGNI
}

\author{
O. E. MANDEL
}

Odessa Astronomical Observatory

\begin{abstract}
The photographic magnitude of V 1057 Cyg is estimated on all plates available at the Odessa plate depositary. The light variation is similar to that of FU Orionis.
\end{abstract}

To study light variations in this remarkable object we have used the Odessa plate depository sky photos. In addition, estimations have been referred to; i.e. the ones made by us from the 'blue' prints of the Palomar Atlas and from the plates available at the Abastumani Astrophysical Observatory. About 600 plates in the photographic and photovisual ranges have been used in all. Unfortunately, light minimum is close to the magnitude limit of our plates, so the star is visible only on plates of particularly good quality.

In the figure are given the mean photographic and photovisual light curves of V 1057 Cyg. Gieseking's data (1973) reduced to our system of stellar magnitudes and Meinunger's and Wenzel's estimations (1972) are presented here as well. Before 1969 the star exhibited slow light fluctuations with an amplitude of $\sim 1^{m}$. One cannot make any certain inferences on the periodicity of these fluctuations because of the scarcity of

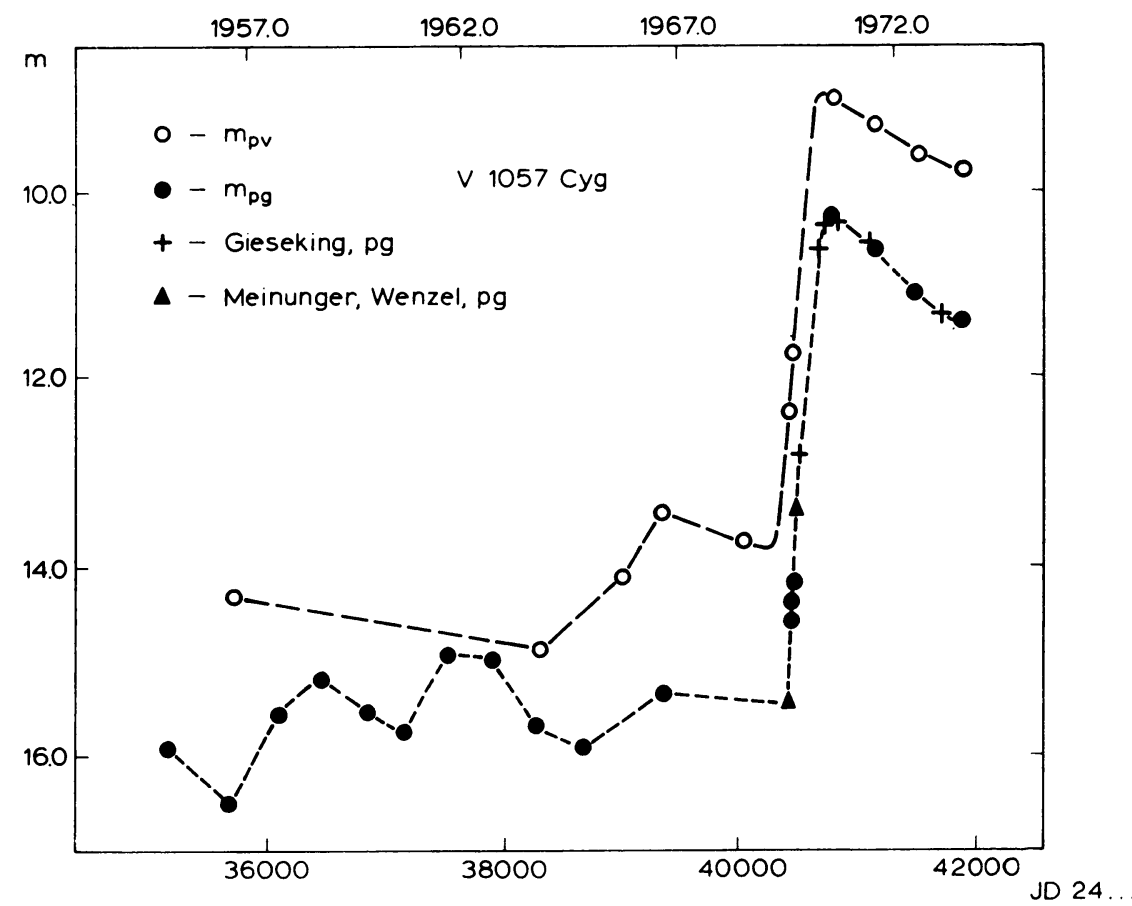

Fig. 1. 
observations. These are rather cyclic variations of the type observed at times in stars of T Tau-type.

A rapid rise in light was observed in July 1969, and the star reached maximum light in late August 1970 . The light increase rate was approximately $0^{m} 01$ and 0.02 per day in photographic and photovisual rays, respectively. The maximum light was followed by slow dimming reaching $m_{\mathrm{pg}} \approx 11.4$ in September 1973 , i.e. $1^{m} \cdot 2$ fainter than maximum. The rate of light decrease is different in different portions of a spectrum: in the photographic region it constitutes 0.001 per day; in the photovisual region it is 0 . 0007 . These variations in light following maximum have been confirmed by photoelectric observations by a number of authors. According to the photoelectric data, the rate of decrease in light in $U$ is 1.5 times greater than that in $B$, and 1.8 times in $V$. Thus, light gradients $\mathrm{d} V / \mathrm{d} B=0.88$ and $\mathrm{d} U / \mathrm{d} B=1.49$ and $\mathrm{V} 1057$ Cyg falls into a region of the gradient diagram far from that populated by $\mathrm{T}$ Tau-type variables for which $\mathrm{d} U / \mathrm{d} B \sim 1.0$ (Kolesnik and Lukatskaya, 1970). Intensive red colour - among other peculiarities in V $1057 \mathrm{Cyg}$ - should be noted at light minimum. At light minimum, the colour index, $m_{\mathrm{pg}}-m_{\mathrm{pv}}$, was about $2^{m}$ on average; on some occasions, it probably amounted to $3^{m}$. At light maximum, $m_{\mathrm{pg}}-m_{\mathrm{pv}}=1.2$.

The V 1057 Cyg light variation character as a whole appears to be similar to that of FU Ori, though there are some distinctions, namely: rate of increase in brightness was greater in FU Ori and the maximum span was considerably shorter in V 1057 Cyg than in FU Ori. The light of V $1057 \mathrm{Cyg}$ has been gradually decreasing for over three years; that of FU Ori remained constant for rather a long time, and the light decrease rate is now on average an order of magnitude slower than in V $1057 \mathrm{Cyg}$.

\section{References}

Gieseking, F.: 1973, Info Bull. Var. Stars, No. 806.

Kolesnik, I. G. and Lukatskaya, F. I.: 1970, Peremennye Zvezdy 17, 224.

Meinunger, L. and Wenzel, W.: 1972, Mitt. Veränd. Sterne 5, No. 9.

\section{REMARKS}

V. P. Tsesevich: The necessity of obtaining long series of observations of RW Aur and/or T Tau-type stars has already been stated. In their monograph The stars of $R W$ Aur type, B. A. Dragomiretskaya and V.P. Tsesevich published more than $\mathbf{4 0 0 0 0}$ estimates of $\mathbf{8 0}$ variables of these types, made from plate collections of Harvard, Odessa and Moscow Observatories. Some of these series covered up to $70 \mathrm{yr}$. Brightness fluctuations over many years were found, among others, for RW Aur, RY Tau, UX Ori, BN Ori, DG Tau, and DF Tau. The stars GZ Aur and FH Aql show rapid, flash-type fluctuations; long-term fluctuations of the 11 yr solar-cycle type were supposed.

The Moscow plate collection included more than 300 plates of the T Tau association. High-luminosity objects seem to have slow variations of small amplitudes; objects with $M$ around $+7^{m}$ to $+8^{m}$ vary rapidly with large amplitudes. Fainter stars $\left(M \sim 10^{m}\right)$ have small-amplitude, flash-type variations.

Abriamashvili (Abastumani) took objective-prism plates with a red filter to obtain the continuum near $\mathrm{H} \alpha$ and the $\mathrm{H} \alpha$ emission. For RW Aur stars, the ratio $I(\mathrm{H} \alpha, \mathrm{em})$ to $I(H \alpha$, cont.) varies between 0 and 8 . Colour indices and absolute magnitudes yield a position at the right side of the main sequence; the closer to the sequence, the larger the ratio mentioned. The non-thermal $\mathrm{H} \alpha$ radiation is enhanced before the star approaches the initial main sequence by gravitational contraction.

A more extensive paper was published by B. A. Dragomiretsk aya (Astron. Circ. U.S.S.R. 824 (1974)). 
Gluzkov: In the Institute of Astrophysics (Alma-Ata) L. N. Kondrat'eva studies spectra of star-like planetary nebulae. She found 4 objects with broad emission lines.

\begin{tabular}{cll}
\hline Object & Halfwidth & Dispersion \\
Sh 2-266 & $5.6 \pm 0.1 \AA$ & 11 and $20 \AA \min ^{-1}$ \\
He 2-446 & $6.6 \pm 0.05$ & 11 and 20 \\
M 1-15 & $8.1 \pm 0.3$ & 20
\end{tabular}

In Sh 266 considerable expansion velocities are observed only in the small nucleus of stellar appearance with the diameter about 9-12". In He 2-446 and M 1-15 the profile consists of two components separated by infrared excesses. The details on the spectra of these objects are given in L. N. Kondrat'eva's paper in Trudy Astrophys. Inst. Acad. Sci. Kazakh SSR 25, 1974 (in press).

A fourth anonymous object was discovered by L. N. Kondrat'eva in 1970 and has characteristics resembling MBV 474=V 1329 Cyg (see Astron. Tsirk., No. 827, 1974): The co-ordinates for 1950 are: $\alpha=18^{\mathrm{h}} 28^{\mathrm{m}} 16^{\mathrm{s}}$. $3, \delta=+2^{\circ} 32^{\prime} 44^{\prime \prime}$. 2. Both objects (the anonymous one and $\mathrm{V} 1329 \mathrm{Cyg}$ ) increased in brightness by about $5^{m}$ and now the brightness remains constant. The spectra of these objects show high degree of excitation and considerable (up to $700 \mathrm{~km} \mathrm{~s}^{-1}$ ) expansion velocities. We may assume that here we observe new type of FU Ori-type objects, that differ from FU Ori and V 1057 Cyg by being connected with very massive $\left(\mathfrak{M} \sim 20 \mathfrak{M}_{\odot}\right)$ and very hot stars. 